\title{
Explorando princípios de Afetibilidade no redesign de aplicações para contextos educacionais
}

\author{
Elaine C. S. Hayashi, Julián E. Gutiérrez Posada, M. Cecília C. Baranauskas \\ Instituto de Computação, UNICAMP \\ \{hayashi, jugutier, cecilia\}@ic.unicamp.br
}

\begin{abstract}
The research that we present in this article concerns the social and affective aspects of interaction in computational systems for learning. Forty students from an undergraduate course have proposed and developed projects that were based on the Design Principles for Affectibility. Such Principles had been proposed with the aim of helping designers to incorporate and explicitly address affective and educational issues in their design practice. The study demonstrates the feasibility of using the Principles for the creation of educational applications with focus on affect, a result that might serve as inspiration to other designers.
\end{abstract}

Resumo. A pesquisa que apresentamos neste artigo está situada no contexto de investigação de aspectos sociais e afetivos em ambientes e sistemas computacionais de apoio à aprendizagem. Quarenta alunos de graduação propuseram e desenvolveram projetos com o objetivo de explorar a aplicação dos Princípios de Design para Afetibilidade. Tais princípios foram propostos para auxiliar designers no processo de incorporar, na prática, questões afetivas e educacionais. O estudo demonstra a viabilidade do uso dos Princípios propostos para criação de aplicações educacionais com foco em afeto; resultado que pode servir de inspiração para outros designers.

\section{Introdução}

O design de tecnologia educacional apresenta desafios particulares ao contexto de aprendizado de crianças. Além dos requisitos de usabilidade e acessibilidade, ao projetar sistemas para o contexto escolar, designers precisam se preocupar com o conteúdo educacional, com conceitos básicos da psicologia e educação infantil, além da preocupação de gerar, durante a interação entre usuários finais e o sistema, respostas afetivas apropriadas para a atividade ou momento. Entendemos afeto como sendo um conceito geral que engloba diversos estados psicológicos relacionados aos valores atribuídos a algo ou alguém, resultando em emoções, sentimentos, preferências, humores, etc. [Ortony et al, 2005]. O afeto tem papel fundamental na vida das pessoas, principalmente no processo de desenvolvimento cognitivo das crianças [LaTaille, 1992]. Estudar afeto em ambientes e sistemas computacionais de apoio à aprendizagem constitui um importante desafio de pesquisa. A principal dificuldade em representar ou tratar de afeto em sistemas educacionais encontra-se na natureza dinâmica e interativa do afeto.

A Computação Afetiva [Piccardi, 1997] entende afeto e emoções como unidades mensuráveis, que primeiramente nascem no indivíduo para então serem entregues para o 
ambiente e para a sociedade. Pesquisas que seguem esta abordagem propõem, com frequência, sistemas computacionais que tentam interpretar as emoções de seus usuários. São os casos dos sistemas de reconhecimento automático de expressão facial, por exemplo. A abordagem interacional [Boehner et al., 2007], contudo, expande essa visão que a computação afetiva tem de algo individual, estático e previsível. A abordagem interacional vê emoções como produto de práticas sociais, resultante de interações dinâmicas, carregadas de valores culturais e ricas em possibilidades de interpretações. É importante conhecer e compreender essas diferentes abordagens em afeto em sistemas computacionais, assim como os desafios que cada uma delas envolve. A visão interacional de afeto demanda processos de design que levem em consideração a diversidade social, cultural e técnica de cada comunidade de usuários.

O conceito de design que, explicitamente, se preocupa com as respostas afetivas das partes interessadas, tanto durante o processo de criação como no uso final do produto de design, é chamado de Design para Afetibilidade [Hayashi e Baranauskas, 2013]. Esse processo de design para Afetibilidade exige novos métodos para criar produtos tecnológicos que, além de estarem em linha com a prática educacional, também façam sentido para a comunidade escolar de usuários finais. Com o intuito de auxiliar designers nesse processo de design para Afetibilidade, foram propostos Princípios de Design para Afetibilidade [Hayashi e Baranauskas, 2013]. Tais princípios tiveram origem em duas frentes paralelas de investigação: uma, de base teórica, que se baseou em trabalhos da psicologia da educação infantil e a outra, de base empírica, que utilizou observações e entrevistas realizadas ao longo de dois anos de imersão em uma escola de ensino fundamental no Brasil.

Os princípios de Design para Afetibilidade são analisados neste artigo, com o objetivo de investigar o potencial de utilizar tais princípios para promover a consideração de aspectos afetivos em projetos (fazer design ou redesign) de tecnologia para o contexto educacional. Quarenta alunos de graduação, atuando como designers de tecnologia educacional, utilizaram os princípios de Design para Afetibilidade para proporem um redesign de aplicações existentes de forma a considerar tais princípios nesse processo. Para tal foram utilizadas aplicações disponíveis na base online do Scratch [Scratch; Maloney et al., 2010]. Neste artigo, apresentamos os resultados preliminares desse trabalho que ilustram o potencial dos princípios no design de aplicações educacionais que considerem aspectos afetivos na/da interação.

Este artigo está organizado da seguinte forma: a Seção 2 apresenta o contexto e metodologia da pesquisa. A Seção 3 explica o conceito de Afetibilidade e seus princípios. Na Seção 4, apresentamos exemplos de uso prático dos princípios na tarefa de redesign de aplicações para suporte ao aprendizado de programação no ensino básico. A Seção 5 mostra os resultados da avaliação das propostas de redesign, que são discutidos na Seção 6. A Seção 7 conclui.

\section{Método, material e participantes}

A realização desta pesquisa contou com a participação dos alunos de disciplina de "Construção de Interfaces Homem-Computador" (IHC), oferecida no primeiro semestre de 2013 como parte do currículo do segundo ano do curso de graduação em "Ciência da Computação" e do terceiro ano do curso de graduação em "Engenharia de Computação". Disciplinas cursadas anteriormente por estes alunos, como por exemplo, "Engenharia de software" e "Projeto de Sistema de Informação", formaram uma base 
para capacitar estes alunos como designers de sistemas.

Na tarefa de validação preliminar dos Princípios de Design para Afetibilidade, os alunos utilizaram o sistema Scratch [Maloney et al., 2010] para trabalhar no redesign de aplicações com propósitos educacionais. Scratch é uma ferramenta interativa criada para permitir que seus usuários possam trabalhar, de maneira colaborativa, com conceitos importantes de matemática e de computação, desenvolvendo o pensamento criativo e sistemático-computacional [Resnick et al., 2009]. O intuito dos criadores do Scratch não foi proporcionar treinamento para preparar profissionais em programação, mas fomentar essa habilidade de pensamento computacional, permitindo que as pessoas possam se expressar por meio da programação. Apesar de ter como público-alvo crianças de 8 a 16 anos, Scratch vem sendo utilizado também por adultos interessados em programação mas que nunca haviam programado antes. Os projetos em Scratch podem ser compartilhados na biblioteca online [Scratch] e outros usuários podem contribuir com seus comentários e desenvolver novos projetos.

Os alunos da disciplina de IHC pesquisaram, nessa base online, por aplicações voltadas ao ensino de programação para crianças. Utilizando os princípios de Design para Afetibilidade, os grupos planejaram e desenvolveram uma nova aplicação em Scratch baseada na primeira, mas incorporando os princípios em seu redesign. Na primeira fase de trabalhos, os grupos apresentaram as aplicações selecionadas, justificando suas escolhas e propondo ideias para a consideração de aspectos afetivos na nova aplicação. Cada grupo indicou como os Princípios de Design seriam aplicados, o que seria mantido e o que seria alterado em cada aplicação Scratch. Na segunda fase, as propostas foram avaliadas por pares na forma de protótipos: cada grupo analisou a proposta de outro grupo, selecionados aleatoriamente, e apresentou, por meio de um formulário específico, seus comentários e sugestões para o trabalho do grupo avaliado. Por fim, na última fase dos trabalhos, os grupos consideraram os comentários recebidos e desenvolveram suas aplicações finais.

Dentro do conjunto de atividades realizadas pelos alunos da disciplina, a atividade de avaliação dos protótipos por pares foi realizada por meio de questionário que considerava cada um dos princípios. Cada princípio foi avaliado em uma escala de zero a cinco, onde o valor cinco representa o melhor valor possível. Além das questões objetivas, uma última pergunta indagava "Se o/a usuário/a alvo da aplicação tivesse que escolher um dos emoticons a seguir para expressar sua satisfação com o design proposto, qual deles você acha que ele/a escolheria?" e apresentava uma matriz de 15 emoticons representando diferentes estados afetivos por meio de faces expressando sorriso, choro, piscada, etc.

Nas próximas seções apresentamos como conduzimos esta atividade com base nos Princípios de Design para Afetibilidade, descrevemos alguns dos projetos desenvolvidos pelos alunos e ilustramos elementos de design que sugerem a efetividade do uso dos princípios nas soluções.

\section{Princípios de Design para Afetibilidade}

Os Princípios de Design para Afetibilidade (PAf) foram propostos como resultado de uma pesquisa composta por duas frentes: uma empírica, derivada de atividades de Observação Participante [Atkinson e Hammersley, 1994], e outra teórica, derivada da análise de textos de autores de renome das áreas de psicologia e educação infantil, nas 
linhas ocidental e oriental (e.g. Piaget, Vigotsky, Walon), com foco nas suas compreensões a respeito do papel do afeto nos processos de aprendizagem. Os princípios são brevemente descritos a seguir. Detalhes em relação ao rationale e outros exemplos de aplicação dos princípios podem ser encontrados em [Hayashi e Baranauskas, 2013].

PAf.1: Permitir a comunicação de estados afetivos. A aplicação deve permitir que usuários possam expressar e interpretar afeto livremente. Abordagens propostas dentro do escopo do Affective Design - Design Afetivo [Picard, 1997] tentam automatizar a comunicação afetiva por meio da tecnologia digital. Diferentemente, o Design para Afetibilidade valoriza e incentiva a livre expressão e interpretação de afeto, rica em significados e possibilidades. Um exemplo de aplicação pode ser encontrado na página do Scratch [Scratch], que permite que usuários se expressem em relação à aplicação exibida, usando o campo de comentários, além dos botões de coração e de estrela.

PAf. 2: Considerar o contexto social do usuário (cultura e valores). O design da aplicação deve ser pensado de forma a refletir elementos da cultura e valores humanos [Friedman e Kahn, 2002; Pereira et al., 2013] importantes para o usuário alvo. Para isso, designers precisam investir em processos de compreensão da realidade vivida por seus usuários. Por exemplo, Kam et al. (2009) sugere jogos para crianças da área rural da Índia que incorporam elementos da cultura da região.

PAf. 3: Possibilitar tailoring. A aplicação deve permitir que os usuários possam ajustar elementos de interação (tailoring [Henderson e Kyng, 1991; Neris e Baranauskas, 2007]), inserindo conteúdo pessoal como mídia (fotos, vídeos, textos), ou conteúdo educacional, de acordo com suas preferências ou especificidade cultural localizadas e que façam sentido dentro do contexto da aplicação desenvolvida. A ferramenta de aprendizagem Ely Doll, por exemplo, permite que os usuários explorem o mundo ao seu redor e tragam para o jogo conteúdos próprios, customizando não apenas a interface como também a interação (e.g. fotos que os usuários tiram se tornam elementos de interação no jogo) [Africano et al., 2004].

PAf. 4: Promover a construção colaborativa. A aplicação deve permitir que usuários possam contribuir uns com os outros, na construção de resultados conjuntos. Entre os colaboradores, além dos próprios alunos, também pais, professores e outros profissionais da educação ou familiares podem ser considerados. Como exemplo, podemos citar a tecnologia para celulares MobileStories que permite que crianças leiam e criem histórias de forma colaborativa [Fails, 2010].

PAf. 5: Promover 'awareness' social. A aplicação deve promover o awareness social dos membros da comunidade de usuários, seja de maneira online ou offline. Por awareness social [Almeida, 2013] entendemos o estado de conhecimento dos usuários sobre a presença ou estado (como localização física, estado emocional/afetivo, atividade sendo conduzida) dos demais pares envolvidos. No jogo para mesa digital proposto por Tanenbaum et al. (2010), por exemplo, a cor da árvore que cada jogador possui indica o quanto o jogador contribuiu para o meio ambiente durante o jogo.

PAf. 6: Explorar a “contaminação" via mídia. Elementos de interação multimídia devem ser explicitamente explorados por designers para construir determinados estados de atenção, motivação ou expectativa nos usuários durante a sua interação com o sistema. Kim et al. (2010), por exemplo, explora uso de diferentes tipos 
de mídia na interface do livro digital que propõe.

A presença dos princípios descritos acima pode ser verificada de forma isolada em diversas aplicações. Enquanto alguns princípios podem parecer óbvios, o objetivo principal da nossa proposta é explicitar a consideração de respostas afetivas no processo de design de interação, fornecendo aos designers um guia simples, porém resultante de um cuidadoso processo de pesquisa [Hayashi e Baranauskas, 2013].

\section{Designers em ação}

Um total de 24 horas-aula dedicadas ao tema do Design para Afetibilidade, mais as horas adicionais de trabalhos em grupos, resultaram em treze propostas de redesign de aplicações para o ensino de programação (considerando como usuários-alvo crianças de 8 a 14 anos de idade). Entre as atividades desenvolvidas durante essas horas estão: Apresentação do "Design para afetibilidade: framework e princípios"; especificação do problema de design; exploração do Scratch em laboratório e escolha de um aplicativo de base; proposta conceitual de design e prototipação; avaliação dos protótipos por pares com instrumento específico; discussão da leitura "How emotion is made and measured" [Boehner et al., 2007]; construção da aplicação com Scratch e apresentação final dos grupos.

Na primeira atividade, o tema afeto em sistemas computacionais foi apresentado, juntamente com o conceito de Afetibilidade, os Princípios de design e o problema de design: "Com base em uma aplicação selecionada da Biblioteca do Scratch, utilizar os princípios do Design para Afetibilidade para criar uma nova aplicação Scratch alinhada aos princípios. Critérios para seleção da aplicação na Biblioteca Scratch: 1. A aplicação selecionada não deve conter elementos propostos nos princípios; 2. A faixa etária do público alvo da nova aplicação [que você irá desenvolver] é 8 a 14 anos.” $\mathrm{Na}$ sequência, atividades em laboratório com computadores permitiram aos alunos navegar pela galeria do Scratch e criar o primeiro documento do grupo, contendo as justificativas para a escolha da aplicação e as propostas de projetos de redesign. Protótipos de baixa fidelidade foram então criados pelos grupos. Os protótipos serviram de base para a avaliação por pares. Para a avaliação, os grupos preencheram formulários criados especificamente para este propósito. Nele, conforme mencionado anteriormente, cada grupo avaliou o uso dos princípios, atribuindo uma nota de 0 a 5 , além de avaliarem outros aspectos, como usabilidade. Os avaliadores também contribuíram com sugestões para melhor aplicação dos princípios, quando necessário. A leitura e discussão do artigo de Boeher et al. (2007) serviu para fortalecer a base teórica dos alunos da disciplina. A compreensão do artigo foi reforçada por meio da discussão em sala de aula e de síntese sobre o artigo, escrita individualmente. Os resultados das avaliações por pares e das apresentações finais são apresentados na Seção 5.

Dentre as treze aplicações resultantes dos trabalhos dos grupos, selecionamos duas para analisar e comentar neste artigo. Para selecionar essas propostas (A1 e A2) dentre as 13 apresentadas, seguimos o critério de maior nota (atribuída ao trabalho da disciplina) dentre as aplicações que efetivamente envolviam ensino de programação uma vez que alguns grupos preferiram trabalhar com outros conteúdos educacionais, como ortografia, expressão artística e conhecimentos gerais.

A primeira aplicação (A1 - RoboProgrammer) exercita o conceito básico de programação (sequência de comandos) por meio do controle dos passos de um 


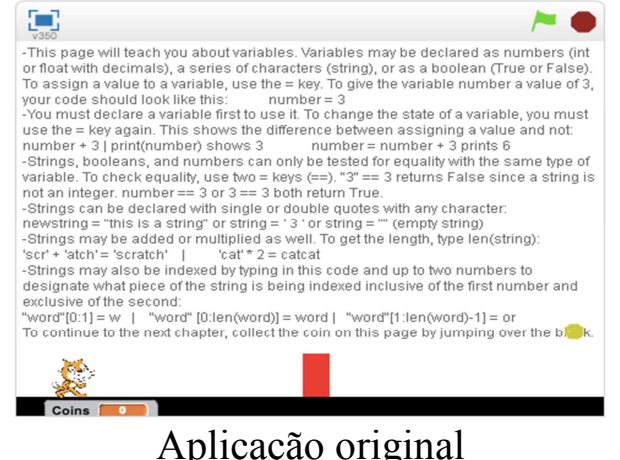

Aplicação original

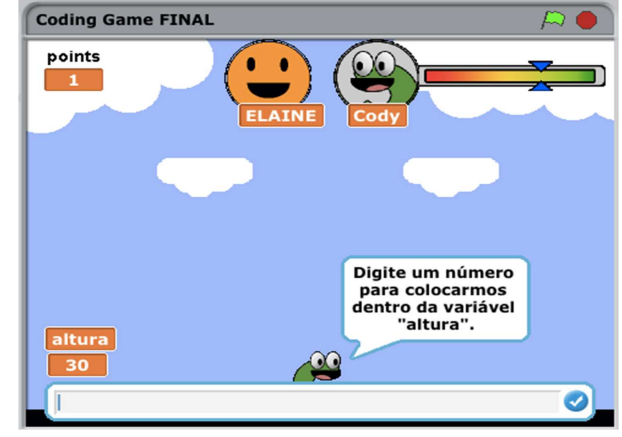

Resultado do redesign

Figura 1 - Telas da aplicação para apoio ao aprendizado de programação, trabalhando o tema 'variáveis': à esquerda, tela da aplicação original. À direita, tela da aplicação final.

personagem. Os alunos propuseram a inclusão de diversos elementos para que os princípios de Design para Afetibilidade pudessem ser evidenciados na interação com a aplicação.

A segunda aplicação selecionada (A2 - Cody) trabalha conceitos da linguagem de programação $\mathrm{C}$ em um jogo no qual o personagem coleta itens, cumpre desafios e responde perguntas. Um dos problemas indicados pelo grupo é o excesso de texto presente na aplicação original. $\mathrm{O}$ grupo propôs remover o excesso de texto, colocar os elementos de programação ilustrados como parte do cenário do jogo (PAf. 6), assim como incluir outras alterações para destacar os princípios na aplicação. A Figura 1 ilustra as diferenças entre a aplicação original e o resultado do redesign de uma das telas da aplicação A2. A Tabela 1 informa algumas das propostas de A1 e A2, evidenciando os Princípios aplicados. Os elementos de interação informados na Tabela 1 não estavam presentes na aplicação original.

Tabela 1 - Aplicações A1 e A2 e alguns dos princípios (PAf.) aplicados.

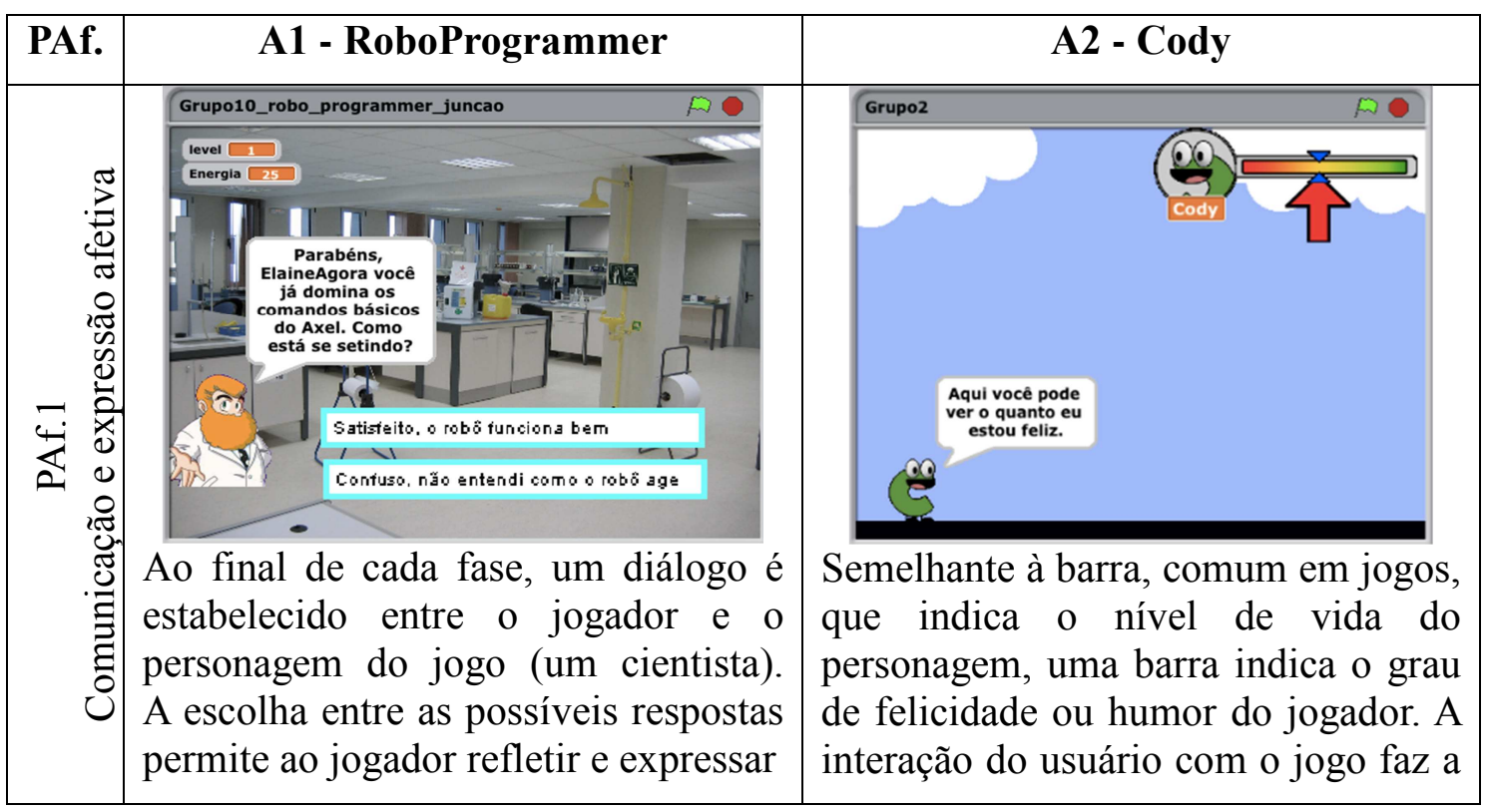




\begin{tabular}{|c|c|c|}
\hline & $\begin{array}{l}\text { sobre a atuação e sentimentos } \\
\text { experienciados durante aquela fase. }\end{array}$ & $\begin{array}{l}\text { barra de humor mudar. Por exemplo, } \\
\text { quando o personagem pergunta se o } \\
\text { jogador está feliz, a barra aumenta ou } \\
\text { diminui conforme a resposta. }\end{array}$ \\
\hline 논 & \multicolumn{2}{|c|}{$\begin{array}{l}\text { As escolhas de design foram feitas com base no usuário alvo: a linguagem } \\
\text { contida nas falas foi adequada segundo o vocabulário e gramática da faixa } \\
\text { etária e os personagens fazem parte das mídias consumidas por eles (desenhos } \\
\text { animados e jogos eletrônicos). }\end{array}$} \\
\hline 赵 & $\begin{array}{l}\text { O enredo do jogo e a perspectiva do } \\
\text { jogador podem ser determinados pelo } \\
\text { próprio jogador, que pode escolher } \\
\text { com qual personagem jogar } \\
\text { (personalidades e objetivos } \\
\text { diferentes). Além disso, as falas usam } \\
\text { o nome informado pelo usuário e a } \\
\text { história se passa na cidade dele. }\end{array}$ & $\begin{array}{llll} & & - & \\
\text { (Princípio não está presente na } \\
\text { solução) }\end{array}$ \\
\hline 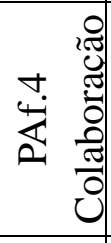 & \multicolumn{2}{|c|}{$\begin{array}{l}\text { Ao final do jogo, os usuários são convidados a compartilhar com outros } \\
\text { jogadores, por meio do forum de discussões da base online do Scratch, suas } \\
\text { soluções e experiências. }\end{array}$} \\
\hline$\because \frac{0}{4}$ & $\begin{array}{l}\text { (Princípio não está presente na } \\
\text { solução) }\end{array}$ & $\begin{array}{l}\text { (Princípio não está presente na } \\
\text { solução) }\end{array}$ \\
\hline 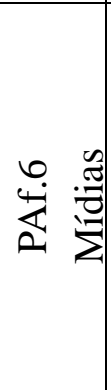 & $\begin{array}{l}\text { Efeitos gráficos e sonoros foram } \\
\text { adicionados para criar o "clima" de } \\
\text { diferentes partes do jogo. Por } \\
\text { exemplo: a música de fundo muda } \\
\text { quando a energia do robô cai ou } \\
\text { quando se depara com uma situação } \\
\text { de perigo; foram adicionados efeitos } \\
\text { gráficos para o momento do ataque. }\end{array}$ & $\begin{array}{l}\text { Quando a barra de humor aumenta, } \\
\text { são acionados efeitos sonoros que } \\
\text { refletem o clima de comemoração. }\end{array}$ \\
\hline
\end{tabular}

\section{Avaliação por pares e resultados}

Na Figura 2, nós podemos apreciar o resultado da avaliação conduzida pelos grupos, para seus pares. Claro que não podemos esquecer que esta é a primeira experiência para todos os grupos no projeto usando os princípios de design para afetibilidade.

A figura 2 mostra os sete primeiros quesitos avaliados, relativos à aplicação dos 6 princípios, mais uma questão sobre usabilidade. Para cada quesito, foi atribuída uma nota de 0 a 5, indicando o quanto o projeto atende esse quesito. As barras indicam a frequência de cada nota para cada quesito. Na figura podemos observar, por exemplo, que houve uma maior aplicação do Princípio 6 ("PAf. 6 Explorar a contaminação via 
mídia), que apareceu em 54\% dos grupos - ou seja, mais da metade dos grupos atribuiu a nota 3 para o uso do Princípio 6. Empatados, 46\% dos grupos atribuíram notas 3, 4 e 5 aos Princípios 1 ("PAf. 1 Permitir a comunicação de estados afetivos"), Princípio 2 ("PAf. 2 Considerar o contexto social do usuário - valores, cultura") e à Usabilidade.

Com relação ao Princípio 4 ("Promover a construção colaborativa"), houve uma distribuição quase uniforme em todas as alternativas, exceto para as notas 4 e 0.

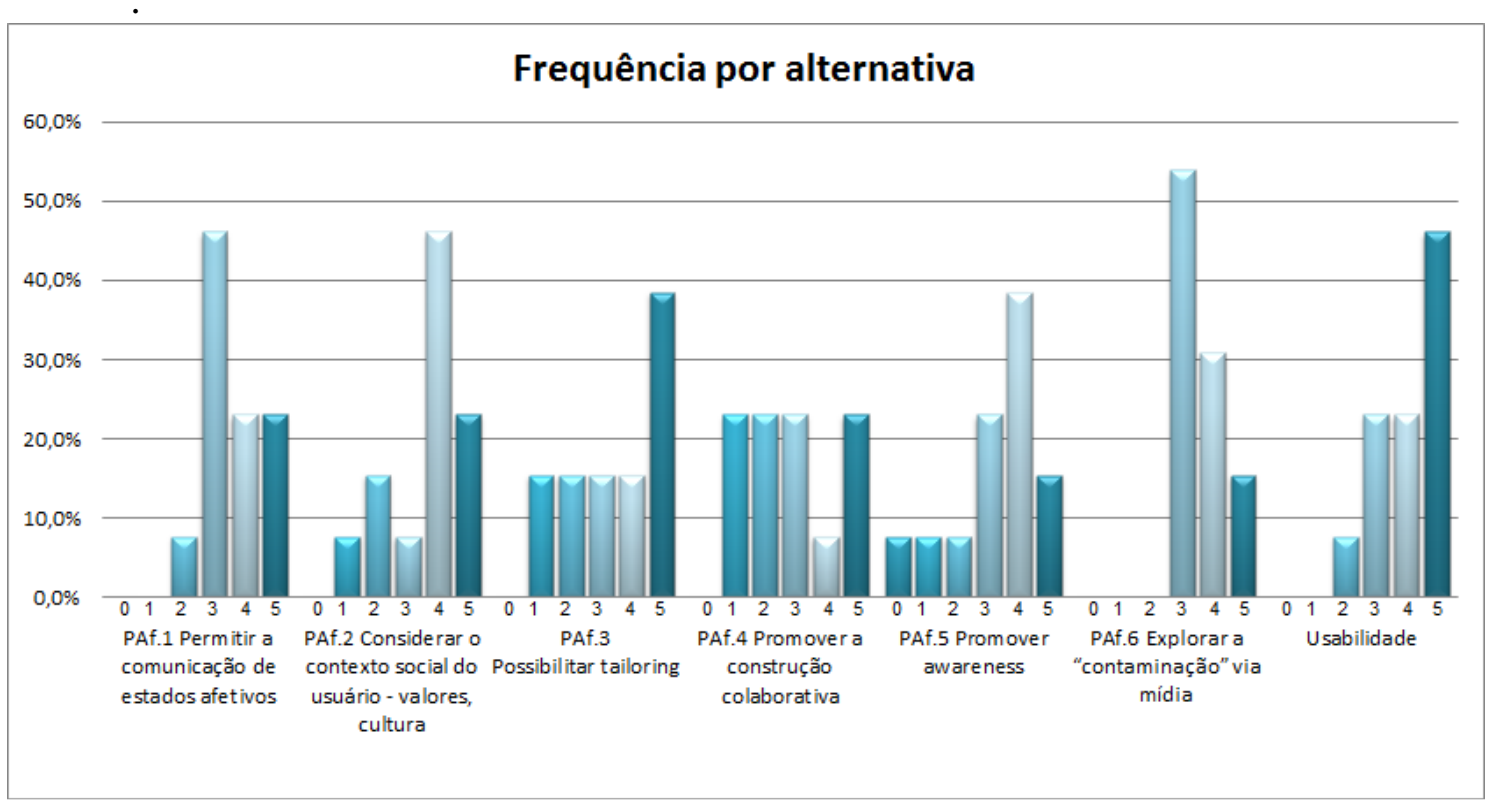

Figura 2: Frequências por alternativa

\section{Discussão e próximos passos}

Em linha com as discussões durante as aulas de condução das atividades de design, o resultado nos indica que o PAf. 6 (uso de diversas mídias para criar atmosferas específicas) foi bastante explorado pelos alunos da disciplina. Apesar de nem sempre ser usado nos projetos de design de interação com o objetivo explícito de ajudar a criar determinadas respostas afetivas, as mídias são comumente trabalhadas nas aplicações educacionais. Contudo, ao compararmos as aplicações originais com os resultados apresentados, podemos notar que os designers das aplicações originais (que não se basearam nos princípios) se preocuparam pouco em explorar tais recursos.

Também em linha com discussões em aula, os resultados indicam que os PAf. 3 (possibilitar tailoring), e PAf. 5 (promover awareness), não foram bem utilizados. Por um lado, existe a limitação de conhecimento dos recursos disponíveis no Scratch. Por exemplo, não se sabia como fazer com que o Scratch se comunicasse com outras aplicações, como com outra aplicação Scratch. Essa restrição dificultou a aplicação do princípio relacionado a awareness social (perceber a presença de outras pessoas). Da mesma forma, a aplicação do princípio relacionado ao conceito de tailoring também pode ter sofrido com as limitações do Scratch. Além disso, os conceitos de tailoring e awareness são conceitos relativamente novos para os alunos da disciplina, quando comparados ao uso de mídias para produção de efeitos, que é encontrado com frequência, especialmente em jogos. Ainda seria necessário verificar como designers mais experientes fariam uso desses princípios em outras plataformas de 
desenvolvimento.

A continuidade desta pesquisa será desenvolvida com a experimentação dos produtos gerados em uma escola de ensino fundamental que vem utilizando o Scratch como ferramenta de aprendizado. Trabalhos anteriores realizados em parceria com esta escola inclui uma atividade intensa de disseminação do Scratch. Nesta atividade, nosso grupo de pesquisa conduziu oficinas com todas as dezenove turmas de alunos, do $1^{\circ}$ ao $9^{\circ}$ ano, em atividades que permitiram que alunos e professores criassem seus primeiros programas, ainda que simples, para terem conhecimento das possibilidades que a ferramenta oferece. Tal iniciativa favorece o desenvolvimento das nossas próximas atividades.

Atuando como parceiros de design [Druin, 2002], os alunos desta escola de ensino fundamental serão convidados a participar na tarefa de avaliar as aplicações Scratch criadas. Esperamos, com isso, poder verificar o uso dos Princípios de Design para Afetibilidade também do ponto de vista do usuário final das aplicações.

\section{Conclusão}

Considerações do aspecto afetivo na interação com sistemas computacionais ainda têm sido pouco explorados na literatura, embora seu valor, especialmente em aplicações educacionais, seja inegável. Este artigo apresentou e discutiu resultados preliminares do uso situado de Princípios de Design para Afetibilidade. Alunos de graduação em Computação no papel de designers propuseram o redesign de aplicações educacionais criadas no Scratch e desenvolveram novas aplicações com base nos princípios apresentados, e tiveram suas propostas avaliadas por pares. Essas atividades constituíram um passo importante no processo de investigação dos princípios. Entretanto, a validação dos princípios de design não se esgota neste trabalho, embora representem um potencial instrumento para pensamos design de interação de sistemas computacionais, especialmente para apoio ao aprendizado. Trabalhos futuros envolvem estender a investigação para incluir usuários finais na avaliação das aplicações geradas, pela perspectiva de seus aspectos afetivos.

\section{Agradecimentos}

Este trabalho é apoiado parcialmente por CNPq (\#160819/2013-0) e CAPES(01-P1965/2012). Agradecemos aos colegas do InterHAD, aos alunos da disciplina de MC750 do $1^{\text {o }}$ semestre de 2013 e à comunidade escolar da EMEF Pde Emílio Miotti.

\section{Referências}

Almeida, L.D.A. (2013) Awareness em redes sociais inclusivas. In: Codesign de Redes Digitais. 1Ed: Editora Penso.

Africano, D., Berg, S., Lindbergh, K., Lundholm, P., Nilbrink, F., \& Persson, A. (2004, April). Designing tangible interfaces for children's collaboration. In CHI'04 extended abstracts on Human factors in computing systems (pp. 853-868). ACM.

Atkinson, P. \& Hammersley, M. (1994) Ethnography and Participant Observation. In Handbook of Qualitative Research, Sage Publications, 1994, pp. 248-261.

Boehner, K., DePaula, R., Dourish, P., \& Sengers, P. (2007). How emotion is made and measured. International Journal of Human-Computer Studies, 65(4), 275-291. 
Druin, A. (2002) The role of children in the design of new technology. Behaviour and Information Technology, 21 (1), pp. 1-25.

Fails, J. A., Druin, A., \& Guha, M. L. (2010, June). Mobile collaboration: collaboratively reading and creating children's stories on mobile devices. In Proceedings of the 9th International Conference on Interaction Design and Children (pp. 20-29). ACM.

Friedman, B.; Kahn, P.H. (2002) Human values, ethics and design. The Humancomputer interaction handbook. L. Erlbaum Associates Inc. Hillsdale, NJ, pp. 11771201.

Hayashi. E. C. S.; Baranauskas, M. C. C. (2013) Design Principles for Affectibility. IC13-17, disponível em: http://www.ic.unicamp.br/ reltech/2013/13-17.pdf (último acesso em Outubro, 2013).

Henderson A; Kyng M (1991) There's no place like home: Continuing Design in Use. In: J.Greenbaum and M. Kyng (eds) Design at work: Cooperative Design of Computer Systems. Hillsdale, NJ, Lawrence Erlbaum Ass., p. 219-240.

Kam, M., Mathur, A., Kumar, A., \& Canny, J. (2009, April). Designing digital games for rural children: a study of traditional village games in India. In Proceedings of the SIGCHI conference on Human factors in computing systems (pp. 31-40). ACM.

Kim, M., Yoo, K. H., Park, C., \& Yoo, J. S. (2010). Development of a digital textbook standard format based on XML. In Advances in Computer Science and Information Technology (pp. 363-377). Springer Berlin Heidelberg.

LaTaille, Y. D. (1992). Desenvolvimento do juízo moral e afetividade na teoria de Jean Piaget. Piaget, Vygotsky, Wallon: teorias psicogenéticas em discussão, 47-73, São Paulo, Summus Editorial.

Maloney, J.; Resnick, M.; Rusk, N.; Silverman, B.; Eastmond, E. (2010) The Scratch Programming Language and Environment. ACM Transactions on Computing Education, Vol. 10, No. 4.

Neris, V. P. A.; Baranauskas, M. C. C. (2007) End-user Tailoring: a Semiotic-informed Perspective. In: Proc. of ICOS 2007), p. 47-53.

Ortony, A., Norman, D.A. \& Revelle, W. (2005) Affect and Proto-affect in effective functioning. Who Needs Emotions? The Brain Meets the Machine. New York: Oxford University Press, pp. 173-202.

Picard, R. (1997) Affective Computing. MIT Press.

Resnick, M., Maloney, J., Monroy-Hernández, A., Rusk, N., Eastmond, E., Brennan, K., ... \& Kafai, Y. (2009). Scratch: programming for all. Communications of the ACM, 52(11), 60-67.

Scratch. http://scratch.mit.edu/explore/?date=this_month (ultimo acesso em Ago/2013).

Tanenbaum, J., Tanenbaum, K., El-Nasr, M. S., \& Hatala, M. (2010). Authoring tangible interactive narratives using cognitive hyperlinks. Proc. of the Intelligent Narrative Technologies III Workshop, pp.6. 